\title{
Video Article \\ Effects of Blast-induced Neurotrauma on Pressurized Rodent Middle Cerebral Arteries
}

\author{
Uylissa A. Rodriguez ${ }^{1,2}$, Yaping Zeng ${ }^{1,2}$, Margaret A. Parsley ${ }^{1,2}$, Bridget E. Hawkins ${ }^{1,2}$, Donald S. Prough ${ }^{1,2}$, Douglas S. DeWitt ${ }^{1,2}$ \\ ${ }^{1}$ Charles R. Allen Research Laboratories, Department of Anesthesiology, University of Texas Medical Branch \\ ${ }^{2}$ The Moody Project for Translational Traumatic Brain Injury Research, University of Texas Medical Branch
}

Correspondence to: Uylissa A. Rodriguez at uarodrig@utmb.edu

URL: https://www.jove.com/video/58792

DOI: doi: $10.3791 / 58792$

Keywords: Neuroscience, Issue 146, TBI, blast-induced traumatic brain injury (bTBI), blast-induced neurotrauma, primary blast injury, cerebral vascular reactivity, middle cerebral arteries (MCA), myogenic response

Date Published: 4/1/2019

Citation: Rodriguez, U.A., Zeng, Y., Parsley, M.A., Hawkins, B.E., Prough, D.S., DeWitt, D.S. Effects of Blast-induced Neurotrauma on Pressurized Rodent Middle Cerebral Arteries. J. Vis. Exp. (146), e58792, doi:10.3791/58792 (2019).

\section{Abstract}

Though there have been studies on the histopathological and behavioral effects of blast exposure, fewer have been dedicated to blast's cerebral vascular effects. Impact (i.e., non-blast) traumatic brain injury (TBI) is known to decrease pressure autoregulation in the cerebral vasculature in both humans and experimental animals. The hypothesis that blast-induced traumatic brain injury (bTBI), like impact TBI, results in impaired cerebral vascular reactivity was tested by measuring myogenic dilatory responses to reduced intravascular pressure in rodent middle cerebral arterial (MCA) segments from rats subjected to mild bTBI using an Advanced Blast Simulator (ABS) shock tube. Adult, male Sprague-Dawley rats were anesthetized, intubated, ventilated and prepared for Sham bTBI (identical manipulation and anesthesia except for blast injury) or mild bTBI. Rats were randomly assigned to receive Sham bTBI or mild bTBI followed by sacrifice 30 or 60 min post-injury. Immediately after bTBI, righting reflex $(R R)$ suppression times were assessed, euthanasia at the time points post-injury was completed, the brain was harvested and the individual MCA segments were collected, mounted and pressurized. As the intraluminal pressure perfused through the arterial segments was reduced in $20 \mathrm{mmHg}$ increments from 100 to $20 \mathrm{mmHg}$, MCA diameters were measured and recorded. With decreasing intraluminal pressure, MCA diameters steadily increased significantly above baseline in the Sham bTBI groups while MCA dilator responses were significantly reduced $(p<0.05)$ in both bTBI groups as evidenced by the impaired, smaller MCA diameters recorded for the bTBI groups. In addition, RR suppression in the bTBI groups was significantly $(p<0.05)$ higher than in the Sham bTBI groups. MCA's collected from the Sham bTBI groups exhibited typical vasodilatory properties to decreases in intraluminal pressure while MCA's collected following bTBI exhibited significantly impaired myogenic vasodilatory responses to reduced pressure that persisted for at least $60 \mathrm{~min}$ after bTBI.

\section{Video Link}

The video component of this article can be found at https://www.jove.com/video/58792/

\section{Introduction}

Similar to that resulting from impact (i.e., non-blast) TBI, blast-induced traumatic brain injury (bTBI) has been associated with cerebral vascular injury $^{1}$ and impaired cerebral vascular compensatory responses to occurrences like alterations in the partial pressures of carbon dioxide $\left(\mathrm{PaCO}_{2}\right)^{2,3,4}$ and oxygen $\left(\mathrm{PaO}_{2}\right)^{5}$. Additionally, blast exposure has caused cerebral arterial vasospasm in animals ${ }^{6}$ and bTBI patients ${ }^{7,8}$. While clinical $\mathrm{TBI}^{9}$ and fluid-percussion injury (FPI ${ }^{10,11,12}$ are associated with impaired cerebral vascular responses to changes in arterial blood pressure (i.e., pressure autoregulation) ${ }^{9,10,11,12}$, uncertainties remain concerning the effects of bTBI on cerebral vascular pressure autoregulation capacity.

The cerebral circulation reacts to variations in systemic arterial pressure with the intent of maintaining a continuous oxygen and nutrient supply delivered to the metabolically active brain ${ }^{13,14,15,16}$. A unique type of homeostasis, autoregulation ${ }^{17,18,19}$ occurs when "an organ maintains a constant blood flow despite changes in blood (perfusion) pressure or other physiologic or pathologic stimuli" ${ }^{20}$. Cerebral arteries constrict or dilate in response to variations in blood pressure, nitric oxide (NO), blood viscosity, $\mathrm{PaCO}_{2}$ and $\mathrm{PaO}_{2}$, etc. ${ }^{4,11,16,21}$. Arterial myogenic response refers to such contractions or dilations. The myogenic vascular response, first described by Bayliss ${ }^{22}$ and a major mechanism contributing to autoregulation of $\mathrm{CBF}$, is characterized by vasoconstriction if perfusion pressure increases and vasodilatation if perfusion pressure decreases $^{14,17}$. This vascular response is the inherent ability of contractile tissues (such as vascular smooth muscle cells, VSMC's) to respond to stretch and/or changes in lumen and/or wall tension ${ }^{23,24,25,26,27,28,29}$. When arteries are stretched (e.g., during intravascular pressure increases), VSMC's constrict $^{24,25,26,28}$

Studies that examine resistance vessels ex vivo have commonly employed one of two methods for testing the pharmacological and physiological properties of isolated resistance vessels: the ring-mounted method and the cannulated, pressurized method. The ring-mounted vessel preparation method involves two wires passed intraluminally through the vessel segment, which hold the segment in place. Measuring the amount of force applied on the isometrically sustained wires gauges the stimulation of the VSMC's. However, this technique carries with it certain reservations, most notably, the inevitable damage sustained by the endothelial layer of the lumen as the wires are passed through it ${ }^{30}$ and the 
varying degree of stretching sustained by the isolated segment which in turn leads to vessel wall distension, ultimately affecting the vessel's sensitivity to pharmacological agents ${ }^{31}$. The cannulated, pressurized vessel preparation methodology utilizes an arteriograph comprised of two separate chambers that each house the placement of a middle cerebral arterial (MCA) harvested from a single animal. A micropipette is inserted into each end of the segment, the proximal end of the segment is fastened to the micropipette with sutures and the lumen is softly perfused with a physiological salt solution (PSS) in order to eliminate blood and any other substances. The distal end is then secured with sutures. Transmural or luminal pressure is set by raising the two reservoirs attached to each pipette to a suitable height above each segment but at different heights with respect to the other ${ }^{32,33,34,35,36}$. Pressure transducers positioned along the reservoirs and micropipettes provide perfusion pressure measurements while vessels are magnified using an inverted microscope outfitted with a monitor, video camera and scaler allowing for measurement of the external MCA diameters. Though both methods are valuable, the cannulated, pressurized vessel preparation methodology better mimics and permits the vessels investigated to be closer to their in vivo conditions ${ }^{32,37}$.

The effects of different types of impact (i.e., non-blast) TBI on cerebral vascular responses have previously been studied in cerebral arterial segments $^{21,35,36,38}$. Using a similar ex vivo MCA protocol for vessel collection, mounting and perfusion as described in the current study, earlier studies obtained success with their respective investigations into the associated mechanisms of cerebral vasculature dysfunction following TBI. Golding et al. ${ }^{34}$ examined endothelial-mediated dilations in adult, male Long-Evans rat MCA's following severe TBI through controlled cortical impact (CCI) injury. In a second study, Golding et al ${ }^{36}$ investigated cerebrovascular reactivity to hypotension or $\mathrm{CO}_{2}$ after harvesting $\mathrm{MCA}^{\prime} \mathrm{s}$ from rats that sustained a mild $\mathrm{CCl}$. Yu et al. ${ }^{38}$ analyzed whether peroxynitrite scavengers improved dilatory responses to reduced intravascular pressure in adult, male Sprague-Dawley rat MCA segments subjected to FPI while Mathew et al. ${ }^{21}$ studied myogenic responses to hypotension in MCA's harvested after moderate, central FPI.

To better investigate the hypothesis that bTBI, like non-blast TBI, results in impaired cerebral vascular reactivity, we tested a mechanism contributing to compromised autoregulation by measuring myogenic dilatory responses to reduced intravascular pressure ex vivo in isolated, pressurized rodent MCA segments (Figure 1) collected from rats subjected to mild bTBI using an Advanced Blast Simulator (ABS) shock tube model (Figure 2 and Figure 3) (see Rodriguez et al. ${ }^{39}$ Table 1) that uses compressed air delivered directly to a driver chamber to generate Freidlander-like ${ }^{40}$ over- and under-pressure waves (see Rodriguez et al. ${ }^{39}$ Figure 1A).

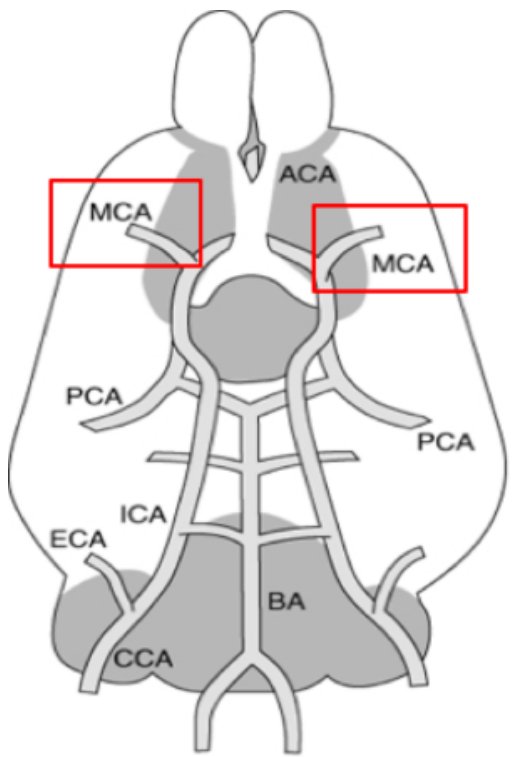

Figure 1: Location of middle cerebral arteries (MCA). Ventral view of the rat brain highlighting the location of the MCA's relative to the posterior cerebral arteries (PCA), internal carotid arteries (ICA), external carotid arteries (ECA), basilar artery (BA) and common carotid arteries (CCA). Please click here to view a larger version of this figure.

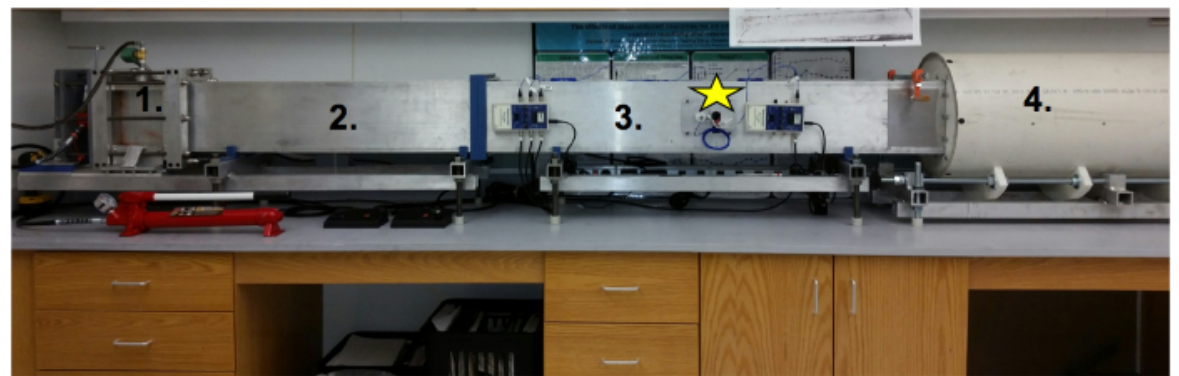

Figure 2: Advanced Blast Simulator (ABS) shock tube device. The ABS used to produce primary blast injury in all study animals. $1=$ driver chamber; 2 = expansion chamber; 3 = specimen chamber; 4 = reflected wave suppressor; yellow star = specimen tray. Please click here to view a larger version of this figure. 


\section{Protocol}

All experimental protocols were approved by the Institutional Animal Care and Use Committee (IACUC) of the University of Texas Medical Branch, an Association for Assessment and Accreditation of Laboratory Animal Care (AAALAC) accredited facility.

\section{Animal Preparation for ABS Blast Injury}

1. Turn on mechanical rodent volume ventilator and set breath rate between $40-45$ breaths per min.

2. Switch thermostatically controlled warming blanket $\mathrm{ON}$ and tape a blue pad over it.

3. Attach ventilator hose to the ventilator.

4. Gather endotracheal intubation sled, laryngoscope, long pickup tweezers, stylet, endotracheal tube, and a cotton swab soaked with $0.05 \mathrm{~mL}$ of $1 \%$ Lidocaine $\mathrm{HCl}$. Organize on the blue pad.

5. Confirm that rodent anesthetic "bubble" chamber, ventilator, air chamber and isoflurane chamber hoses are securely connected and attached to their respective plug or socket. The anesthetic bubble chamber clamp should be OPEN; the ventilator clamp should be CLOSED.

6. On the air chamber, set the knob for room air to $2 \mathrm{~L} / \mathrm{min}$ and the knob for oxygen to $1 \mathrm{~L} / \mathrm{min}$.

7. Turn on the isoflurane and set the knob to $4 \%$ of volume mixture.

8. Start a timer and place a young adult ( $\approx 3$ months old), male Sprague-Dawley rat $(350-400 \mathrm{~g})$ in the anesthetic bubble chamber for 4-6 min.

9. Weigh rat at the 2 min mark.

10. Confirm rat is fully anesthetized by gently pinching hind paw toes. If no paw withdrawal is observed, reduce the room air and set knob to $1 \mathrm{~L} /$ $\min$, oxygen to $0.4 \mathrm{~L} / \mathrm{min}$ and isoflurane to $2 \%$ of volume mixture.

11. Turn on rectal telethermometer temperature monitor.

12. OPEN the clamp to the ventilator and CLOSE the clamp to the anesthetic bubble chamber.

13. Remove rat from anesthetic bubble chamber and position on endotracheal intubation sled.

14. Intubate rat. Place laryngoscope in animal's mouth, use long pickup tweezers to position the tongue out of the way, swab lidocaine-soaked cotton swab tip along inside of the throat and gently insert stylet containing the endotracheal tube into rat's trachea.

15. Once intubated, insert the end of the ventilator hose to the outside end of endotracheal tube and observe and confirm the rat is breathing steadily and without difficulty.

16. Tie down the endotracheal tube into place taking care that the tongue is free from the knot.

17. Apply white petroleum jelly to rectal telethermometer probe and insert directly beneath the tail.

18. Remove the ABS specimen tray from the specimen chamber and place under the heat lamp for warming before rat placement on tray.

19. Shave the top of the rat's scalp starting above the eyes and down to between the ears.

20. Cut a standard-sized foam ear plug into identical halves with scissors. Start at the center of the base of the plug and cut straight up to the rounded tip. Insert a halved piece into each ear tip first along the ear canal until contact is made with the tympanic membrane.

21. Monitor rectal temperature. Once a temperature of $37^{\circ} \mathrm{C}$ is reached, the rat is ready to be loaded into the ABS specimen tray.

22. Secure the rat onto the ABS specimen tray. Remove the ventilator hose from the endotracheal tube and quickly but softly slide the rat into the top end of the tray, gently guiding the head through the head holder opening and rubber collar. Reinsert the ventilator hose back into the endotracheal tube, check the rubber collar to make sure it is securely but not tightly around the neck and verify that the rat is laying in a lateral prone position (Figure 3).

23. Turn off the isoflurane and remove the ventilator hose from the endotracheal tube.

24. Lock and secure the ABS specimen tray containing the anesthetized rat into the ABS specimen chamber.

25. Gently pinch hind paw toes using long tweezers every $3 \mathrm{~s}$ until a withdrawal reflex response is elicited.

\section{ABS Blast Device Preparation and Blast-TBI Induction}

NOTE: Protocol steps $2.1-2.10$ are typically completed at the same time as steps $1.1-1.22$ so the ABS is ready for blast injury administration right after the rat is loaded and secured into the specimen chamber.

1. Loosen the hydraulic hand pump (Figure 4A) knob in order to allow for any residual trapped air to escape from the driver chamber (Figure 4B) and to loosen the chamber from its seal.

2. Loosen the cap nuts (Figure 4C) from the all-thread rods (Figure 4D) surrounding the driver chamber and slide the chamber to the left and away from the expansion chamber (Figure $4 \mathbf{E}$ ).

3. Completely remove the two all-thread rods and their corresponding cap nuts located at the top of the driver chamber in order to allow for placement of the mylar sheets in between the driver and expansion chamber.

4. Stack and tape together along the top edge four pre-cut and pre-measured ( $30 \mathrm{~cm}$ length, $20 \mathrm{~cm}$ width, 0.004 in. thick) mylar sheets (forming a mylar 'membrane') using a $2.54 \mathrm{~cm}$ piece of masking tape. Using a second piece of tape, securely tape the top edge of the mylar membrane to the top of the expansion chamber and over the center of the opening between the driver and expansion chambers (Figure $4 \mathbf{F}$ ).

5. Secure the driver chamber against the mylar membrane by replacing the two all-thread rods at the top of the chamber and hand-tightening all cap nuts surrounding the chamber.

6. Situate the accessory steel block against the hydraulic hand pump block and driver chamber until securely fit.

7. Tighten the hydraulic hand pump knob and confirm the driver chamber remains pressurized with no leaks by observing a constant pressure threshold on the hydraulic gauge.

8. Open the trigger acquisition file that records ABS blast device pressure traces on the ABS blast device computer.

9. Loosen the compressed air tank's (Figure 4G) main knob enough to slightly open the airway.

10. Operate the hydraulic hand pump until the gauge indicator reaches the red arrow indicating a desired chamber pressure level of $\approx 5,000$ psi.

11. Secure and position the anesthetized animal onto the ABS specimen tray (Figure $4 \mathbf{H}$ ) in a lateral prone position (Figure 3 ) and lock the specimen tray into the ABS specimen chamber (Figure 4I).

12. Gently pinch a hind paw using long tweezers every $3 \mathrm{~s}$ until a withdrawal reflex response is elicited. 
13. Click Start on the opened acquisition page on the ABS blast device computer.

14. Once the 'Acquisitioning' window appears on the screen, press and hold down the ABS blast device trigger until the blast goes off, rupturing the mylar membrane and administering the ABS blast injury $(20.9 \mathrm{psi} \pm 1.14,138 \mathrm{kPa} \pm 7.9)$ to the rat. Right after the blast detonates, start a second timer to keep track of how much time (in min and s) has elapsed post-injury.

15. Remove the rat from the ABS specimen tray and return to the blue pad and warming blanket, placing him fully on his back for assessment of righting reflex suppression.

16. Record the time for return of the righting reflex. Observing the second timer, document in min and s the length of time post-injury it takes for the rat to roll from his back onto his stomach three successive times. Return the rat to the anesthetic bubble chamber.

17. Loosen the hydraulic hand pump knob in order to allow for driver chamber loosening and movement.

18. Tighten the compressed air tank's main knob in order to close the airway.

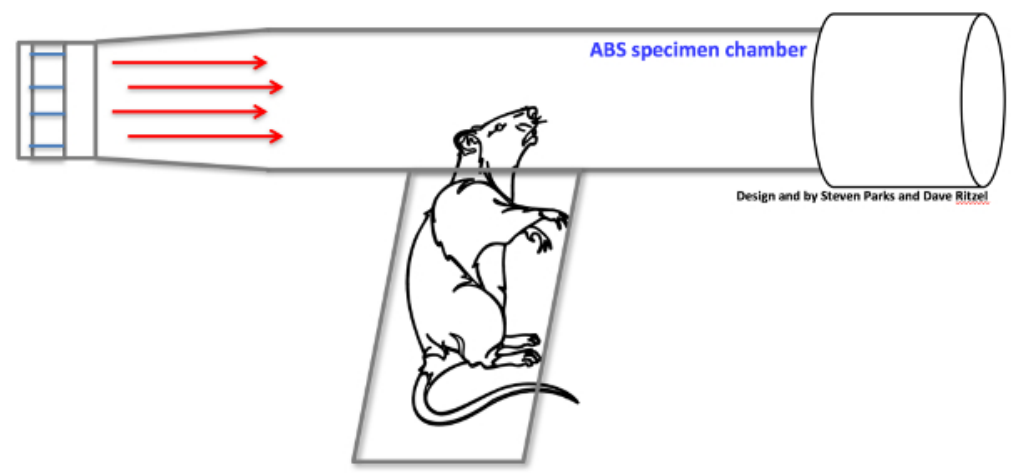

Figure 3: Rat placement on ABS specimen tray and inside ABS. Direction and orientation of the study animal inside the ABS. When placed in the ABS, the animal is in a transverse prone position with the dorsal surface of the head perpendicular to the shock wave direction (red arrows). Please click here to view a larger version of this figure.

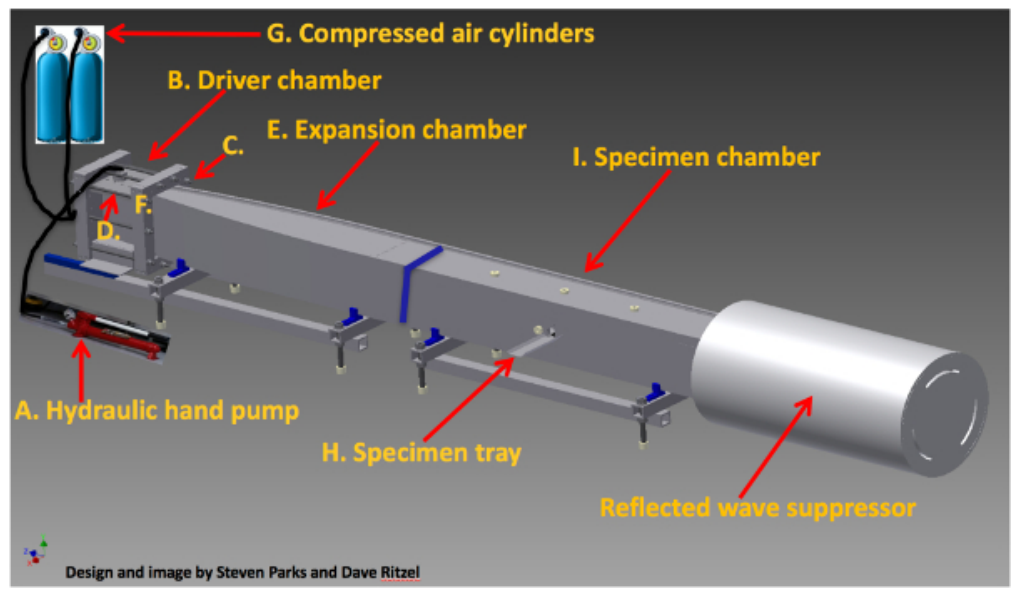

Figure 4: Advanced Blast Simulator (ABS) shock tube device schematic. Major components of the $A B S . A=$ hydraulic hand pump; $B=$ driver chamber; $C$ = cap nuts; $D=$ all-thread rods; $E=$ expansion chamber; $F=$ location of mylar membrane placement; $G=$ compressed air cylinders; $\mathrm{H}$ = specimen tray; I = specimen chamber. Please click here to view a larger version of this figure.

\section{Preparation of Rodent MCA PSS Solution}

NOTE: Protocol steps 3.1-3.3 are typically completed at the same time as steps $1.1-1.22$ to have the PSS solution is ready for use.

1. Prepare and mix a $1,000 \mathrm{~mL}$ physiological salt solution (PSS) of the following composition and concentrations: $130 \mathrm{mM} \mathrm{NaCl} ; 4.7 \mathrm{mM} \mathrm{KCl}$;

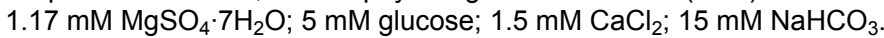

2. Equilibrate the PSS with a gas mixture of $21 \% \mathrm{O}_{2}$ and $5 \% \mathrm{CO}_{2}$ in a balance of $\mathrm{N}_{2}$. The solution is ready when the $\mathrm{pH}$ reads 7.4 . NOTE: All gases are obtained from compressed gas cylinders in the above mentioned concentrations.

3. Fill the reservoir bottles and tubing with prepared PSS solution and chill the remaining solution.

\section{Extraction of Rodent MCA Segments}

1. After documenting the length of the righting reflex time, return the rat to the anesthetic bubble chamber. CLOSE the clamp to the ventilator, and OPEN the clamp to the chamber. Turn the isoflurane on to $4 \%$ of volume mixture and keep the rat in the chamber for $2-3$ min until deeply anesthetized. 
2. Once the rat is anesthetized, OPEN the clamp to the ventilator and CLOSE the clamp to the anesthetic bubble chamber. Reduce the isoflurane to $2 \%$ of volume mixture, and remove him from the bubble chamber. Place him on his stomach on the warming blanket and reinsert the end of the ventilator hose to the outside end of endotracheal tube.

3. Maintain mechanical ventilation at a breath rate between $40-45$ breaths per min and anesthesia at $2 \%$ of volume mixture for either 30 or 60 min immediately post-bTBI injury.

4. After completion of either the 30 or 60 min survival time, increase the isoflurane to $4 \%$ of volume mixture, and deeply anesthetize for $5-6$ min. Immediately euthanize by decapitation using a rodent-specific guillotine.

NOTE: In these experiments, we kept the animals anesthetized and mechanically ventilated following the return of righting reflex until decapitation. If the study requires longer survival timepoints, analgesics should be administered to the animal before emergence from anesthesia.

5. Delicately remove the brain from the skull. Use a \#10 scalpel blade to make a central, 1.5 inch vertical, bone-deep incision from the top of the shaved scalp down to the occipital condyle.

6. Use small bone rongeurs to open and separate the scalp skin from the skull bone.

7. Use large bone rongeurs to cut and extract the occipital, interparietal, and lower half of the frontal bones encasing the brain.

8. Use a surgical spatula to carefully excavate the brain out of the skull once the brain is free of surrounding bone.

NOTE: Take extreme caution when separating the brain from the skull so as not to unnecessarily tug, jerk or pull the delicate MCA segments from the cranial wall.

9. Deposit the harvested brain into the chilled PSS solution contained in a small glass Petri dish that is directly resting on top of a solid ice-block.

10. Carefully remove both the left and right MCA beginning at the Circle of Willis. Continue removing the segment laterally and dorsally for approximately $4-5 \mathrm{~mm}$.

11. Gently clean the collected MCA segments of approximately $4-5 \mathrm{~mm}$ in length of any connective tissue using microforceps.

12. Mount the MCA's on the arteriograph. Cannulate the proximal end of each segment with the first glass micropipette (diameter $\approx 70 \mu \mathrm{m})$ and secure with a 10-0 nylon suture.

13. Softly perfuse the lumina with PSS to remove any residual blood and other contents from the lumen.

14. Cannulate the distal end of each segment with the second micropipette without stretching the MCA segment and secure with a 10-0 nylon suture.

15. After successful mounting of the MCA segment, place the chamber on top of an inverted microscope's stage for magnification of the vessels. The microscope is equipped with a video camera, monitor and a video scaler calibrated with an optical micrometer for arterial diameter measurements.

16. Fill each segment and the surrounding arteriograph bath with continuously circulated PSS warmed from room temperature to $37{ }^{\circ} \mathrm{C}$ and equilibrated with the gas mixture of $21 \% \mathrm{O}_{2}$ and $5 \% \mathrm{CO}_{2}$ in a balance of $\mathrm{N}_{2}$.

17. Equilibrate the MCA segments at a pressure of $50 \mathrm{mmHg}$ for $60 \mathrm{~min}$ by raising the reservoir bottles connected to the micropipettes to an appropriate height above the segments.Pressure transducers located between the micropipettes and reservoir bottles will assess transmural pressure within the MCA segment indicating when the desired $50 \mathrm{mmHg}$ pressure is attained.

18. After the conclusion of the equilibration period, increase the intravascular pressure to $100 \mathrm{mmHg}$ by setting the reservoir bottles at different heights.

19. Deliver $30 \mathrm{mM} \mathrm{K}^{+}$(for confirmation of vessel contraction) via the luminal perfusate and measure arterial diameters. Approximately 10 min later deliver $10^{-5} \mathrm{M}$ Ach (for vessel dilation) and measure arterial diameters.

20. Examine vessel dilatory responses. Lower the reservoir bottles to reduce the intravascular pressure from $100 \mathrm{mmHg}$ to $80 \mathrm{mmHg}$. Allow the MCA segments to equilibrate for $10 \mathrm{~min}$. Measure arterial diameters.

21. Reduce the intravascular pressure from $80 \mathrm{mmHg}$ to $60 \mathrm{mmHg}$. Allow the MCA segments to equilibrate for $10 \mathrm{~min}$. Measure arterial diameters.

22. Reduce the intravascular pressure from $60 \mathrm{mmHg}$ to $40 \mathrm{mmHg}$. Allow the MCA segments to equilibrate for $10 \mathrm{~min}$. Measure arterial diameters.

23. Reduce the intravascular pressure from $40 \mathrm{mmHg}$ to $20 \mathrm{mmHg}$. Allow the MCA segments to equilibrate for $10 \mathrm{~min}$. Measure arterial diameters.

\section{Representative Results}

Mean bTBI overpressure for all study animals was $20.9 \mathrm{psi} \pm 1.14(138 \mathrm{kPa} \pm 7.9)$. The mean duration of righting reflex (RR) suppression for rats subjected to ABS bTBI shockwave exposure ( $5.37 \mathrm{~min} \pm 2.1)$ was not significantly longer $(p=0.36$, bTBI vs. sham) than in the sham group (5.10 $\min \pm 1.6)$.

In both the 30 and 60 min sham groups, MCA diameters increased above baseline as intraluminal pressure was reduced from 100 to $20 \mathrm{mmHg}$. Compared to their corresponding sham groups, the MCA dilatory responses to the continuous imposed reduction in intravascular pressure in the observed $30 \mathrm{~min}(p=0.01$, bTBI vs. sham) and $60 \mathrm{~min}(p=0.02$, bTBI vs. sham) ABS bTBI groups were significantly reduced after blast exposure (Figure 5). For a more detailed discussion of these results, see Rodriguez et al. ${ }^{39}$.

These studies revealed that mild bTBI significantly impaired cerebral compensatory dilator responses to reduced intravascular pressure in MCA segments 30 and 60 min after mild bTBI while the mild shock wave levels used in these studies resulted in durations of suppression of RR (<30 s) similar to those in sham-injured rats.

Statistical analyses were performed with software. The myogenic response to changes in intravascular pressure was assessed by calculating percent change from baseline $(100 \mathrm{mmHg})$ for each level of intraluminal pressure $(80,60,40$, and $20 \mathrm{mmHg})$. Unpaired Student's t-tests were used to evaluate differences between the bTBI and sham group baselines. Differences in MCA dilator responses between bTBI and sham groups were assessed using a repeated one-way analysis of variance (ANOVA) Dunnett's multiple comparisons test and a Bartlett's test for equal variance. 
Due to the reduction in statistical power that results from repeated testing, comparisons at each specific pressure point in the MCA experiments (e.g., between 100 and $80 \mathrm{mmHg}$ or between 60 and $40 \mathrm{mmHg}$, etc.) were not conducted. Significance was accepted at the $p \leq 0.05$ level. All data in the text, referenced table, and figure is expressed as means \pm standard errors of the means (SEM).

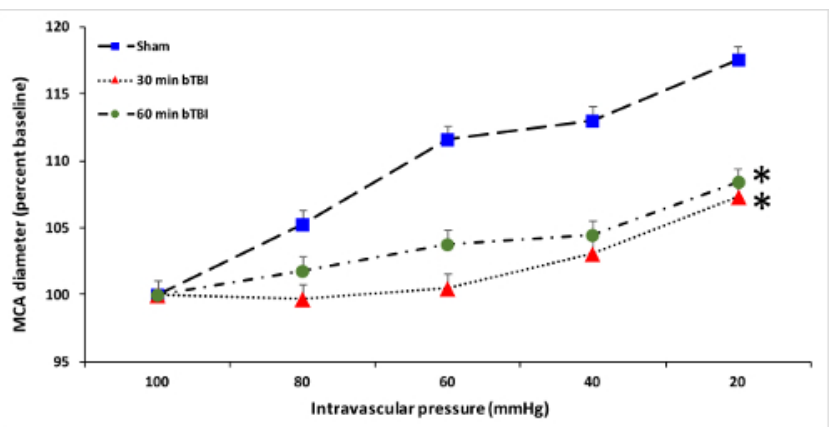

Figure 5: Effects of bTBI on middle cerebral arterial (MCA) responses to reduced intravascular pressure. Dilator responses to progressive reductions in intravascular pressure exhibited impaired vasodilatory responses and were significantly reduced in the $30 \mathrm{~min}(p=0.01, \mathrm{bTBI} v \mathrm{~s}$. sham) and $60 \mathrm{~min}(p=0.02$, bTBI vs. sham) bTBI groups $(n=6 /$ group $)$ after blast exposure compared to both Sham groups $(n=12)$. In both the 30 and 60 min sham groups, MCA diameters increased above baseline as intraluminal pressure was reduced from 100 to $20 \mathrm{mmHg}$. Values are plotted as means \pm SEM. ${ }^{*} p<0.05$ vs. sham. Please click here to view a larger version of this figure.

\section{Discussion}

As with all protocols and instructions, it is imperative that certain steps for the protocol in this particular study are followed as accurately and as precisely as possible. After the initial intubation of the rat it is important to confirm that it is breathing steadily and without difficulty. Mistakenly inserting the endotracheal tube into the esophagus instead of the trachea will result in rasping, difficult breaths, bleeding and the subsequent rousing of the rat due to deficient anesthetic delivery to the lungs.

When taping the mylar membrane sheets over the center of the opening between the driver and expansion chamber, it is imperative that the sheets are centered and cover the entire opening ${ }^{39,41}$. Misaligning the sheets over the opening will result in air leakage from the driver chamber, a drop in the required pressure for membrane burst-potential and denial of administration of the blast injury. Properly situating and securely fitting the accessory steel block against the hydraulic hand pump block and driver chamber is also essential as is tightening the hydraulic hand pump knob and confirming the driver chamber remains pressurized without leaks. Proper placement of the steel block allows for the driver chamber to tightly close against the expansion chamber, thus creating the mandatory seal required over the chamber opening by the Mylar membrane sheets and between the driver and expansion chamber.

During preparations before the MCA vessel extractions, gassing the PSS with the requisite mixture of $21 \% \mathrm{O}_{2}$ and $5 \% \mathrm{CO}_{2}$ in a balance of $\mathrm{N}_{2}$ equilibrates the solution and facilitates the necessitated neutral physiological pH needed for a working PSS solution ${ }^{21,33,34}$.

Equilibrating the segments at a constant pressure for $60 \mathrm{~min}^{21,32,33,34}$ is extremely obligatory as this step permits the segments to constrict following a maximum dilation displayed during their first primary pressurization. This event demonstrates the occurrence of spontaneous tone, a property suggestive of a healthy artery ${ }^{32,33,34}$. Though assorted pressure levels for segment equilibration have been utilized in other studies $^{33,34,42}$, this study and those of Mathew et al. ${ }^{21}$, Golding et al. ${ }^{35}$ and Golding et al. ${ }^{43}$ equilibrated the segments at $50 \mathrm{mmHg}$. While equilibrating collected segments anywhere between $40 \mathrm{mmHg}-100 \mathrm{mmHg}^{32}$ allows for some flexibility and modification for that step of the protocol, an hour equilibration period within those pressure parameters ultimately confirms healthy arteries needed for continuation of the experiment.

Taking extreme caution when removing the brain from the skull and the left and right MCA segments from the Circle of Willis while keeping those vessels intact is perhaps the most critical step of the entire protocol. Puncturing the brain with the bone rongeurs, tearing or severe stretching of the segments during removal or accidentally pulling the vessels with the surgical spatula when excavating the brain out of the skull will ultimately result in destruction of the harvested MCA's, causing unserviceable segments and discontinued use of that set of arteries, ultimately voiding the entire experiment for that animal.

Though measuring cerebral vascular responses to dilatory or constrictory stimuli in MCA segments ex vivo collected after impact or blast TBI in vivo has yielded success, the methodology is not without its difficulties and/or limitations. Perhaps one of the more discernable complexities linked with examining the consequences of TBI on the circulation of the cerebral vasculature is detaching the explicit effects of TBI on the vessels from the implicit effects incurred due to the various materials and elements generated by the injured brain ${ }^{44}$. This conceivable perplexity can potentially be evaded by analyzing ex vivo the vasoconstrictory and vasodilatory reactions of harvested, perfused and/or pressurized MCA's. In an effort to reduce the duration of time that cerebral arteries in vivo are exposed to locally discharged parenchymal vasoactive material prior to death, collection of the cerebral arteries directly after TBI can lessen the degree of such prolonged-exposure effects. Ex vivo studies on isolated MCA's additionally present the prospect of analyzing mechanisms of traumatic vascular injury through the use of particular receptor agonists and antagonists or reputed vehicles of vascular injury that would not afford scrutiny as efficiently or as discriminatory in vivo. Subsequently, this ex vivo method can be combined with ex vivo exposure to drugs to test resulting myogenic responses (vasoconstriction or dilation of vessel segment due to intravascular or extravascular drug exposure).

Other limitations include roughly or impatiently removing the MCA's from the harvested brain which can result in premature tearing of the vessels, thus voiding their use. In addition, letting more than a few minutes elapse between euthanasia of the animal, collection of the vessels and their placement in the prepared PSS solution can also negate their viability. When properly performed and followed, the methods described 
in this protocol for testing myogenic responses of MCA's after bTBI takes several hours from start to finish and attempts at curtailing the length of time required for success can result in experimental failure. However, this method is done in vitro and utilizes considerably more costeffective instrumentation and equipment than in vivo high-resolution magnetic resonance (MR) imaging ${ }^{45,46}$ or conventional Doppler sonography/ velocimetric techniques ${ }^{47,48,49}$ which are also employed for vessel studies.

These findings that mild bTBI injury is associated with impaired cerebral dilatory responses to reduced intravascular pressure could potentially be a function of the vasospasm ${ }^{6,7}$ and VSMC hyperconstriction ${ }^{50}$ previously reported after blast exposure ultimately leading to occurrences such as reduced relative cerebral perfusion. Additionally, blast-induced damage hampering normal dilatory reactions of the cerebral vasculature could possibly promote further reductions in cerebral perfusion when combined with arterial hypotension, a frequent incidence during combat operations.

These results indicate that bTBI results in a change to the mechanisms facilitating arterial vascular control. Though acute-phase cerebral vascular impairment of arterial myogenic response to reductions in intravascular pressure for at least an hour post-injury were observed, there remain gaps in information surrounding the acute phase after bTBI. The importance of identifying what physical and biochemical deficiencies injuries to the cerebral vasculature and the brain exposure to bTBI causes could aid in determining the level of therapeutic and/or rehabilitative success fairly immediately after injury.

\section{Disclosures}

The authors have nothing to disclose.

\section{Acknowledgments}

Studies were completed as part of a team supported by The Moody Project for Translational Traumatic Brain Injury Research and award W81XWH-08-2-0132 from the U.S. Army Medical Research and Material Command - Department of Defense.

\section{References}

1. DeWitt, D.S. Prough, D.S. Blast-induced brain injury and posttraumatic hypotension and hypoxemia. Journal of Neurotrauma. 26 (6), 877 887 (2009)

2. Overgaard, J. Tweed, W.A. Cerebral circulation after head injury. Part 1: CBF and its regulation after closed head injury with emphasis on clinical correlations. Journal of Neurosurgery. 41 (5), 531 - 541 (1974).

3. Wei, E.P., Dietrich, W.D., Povlishock, J.T., Navari, R.M., Kontos, H.A. Functional, morphological, and metabolic abnormalities of the cerebral microcirculation after concussive brain injury in cats. Circulation Research. 46 (1), 37 - 47 (1980).

4. Wei, E.P., Kontos, H.A., Patterson, J.L. Dependence of pial arteriolar response to hypercapnia on vessel size. TheAmerican Journal of Physiology. 238 (5), 697 - 703 (1980).

5. Lewelt, W., Jenkins, L.W., Miller, J.D. Effects of experimental fluid percussion injury of the brain on cerebrovascular reactivity of hypoxia and to hypercapnia. Journal of Neurosurgery. 56 (3), 332 - 338 (1982).

6. Bauman, R.A. et al. An introductory characterization of a combat-casualty-care relevant swine model of closed head injury resulting from exposure to explosive blast. Journal of Neurotrauma. 26 (6), 841 - 860 (2009).

7. Armonda, R.A. et al.Wartime traumatic cerebral vasospasm: recent review of combat casualties. Neurosurgery. 59 (6), 1215 - 1225 (2006).

8. Ling, G., Bandak, F., Armonda, R., Grant, G., Ecklund, J. Explosive blast neurotrauma. Journal of Neurotrauma. 26 (6), 815 - 825 (2009).

9. Bouma, G.J., Muizelaar, J.P. Relationship between cardiac output and cerebral blood flow in patients with intact and with impaired autoregulation. Journal of Neurosurgery. 73 (3), 368 - 374 (1990).

10. Lewelt, W., Jenkins, L.W., Miller, J.D. Autoregulation of cerebral blood flow after experimental fluid percussion injury of the brain. Journal of Neurosurgery. 53 (4), 500 - 511 (1980).

11. DeWitt, D.S. et al.Effects of fluid-percussion brain injury on regional cerebral blood flow and pial arteriolar diameter. Journal of Neurosurgery. 64 (5), 787 - 794 (1986).

12. Engelborghs, K. et al. Impaired autoregulation of cerebral blood flow in an experimental model of traumatic brain injury. Journal of Neurotrauma. 17 (8), 667 - 677 (2000).

13. Mchedlishvili, G. Physiological mechanisms controlling cerebral blood flow. Stroke. 11 (3), 240 - 248 (1980).

14. Kontos, H.A. Regulation of the cerebral circulation. Annual Review of Physiology. 43, $397-407$ (1981).

15. Golding, E.M., Robertson, C.S., Bryan, R.M.Jr. The consequences of traumatic brain injury on cerebral blood flow and autoregulation: a review. Clinical and Experimental Hypertension. 21 (4), 299 - 332 (1999).

16. DeWitt, D.S. Prough, D.S. Traumatic cerebral vascular injury: the effects of concussive brain injury on the cerebral vasculature. Journal of Neurotrauma. 20 (9), 795 - 825 (2003).

17. Paulson O.B., Strandgaard, S., Edvinsson, L. Cerebral autoregulation. Cerebrovascular and Brain Metabolism Reviews. 2 (2), 161 - 192 (1990).

18. Lang, E.W., Diehl, R.R., Mehdorn, M. Cerebral autoregulation testing after aneurysmal subarachnoid hemorrhage: the phase relationship between arterial blood pressure and cerebral blood flow velocity. Critical Care Medicine. 29 (1), 158 - 163 (2001).

19. Soehle, M., Czosnyka, M., Pickard, J.D., Kirkpatrick, P.J. Continuous assessment of cerebral autoregulation in subarachnoid hemorrhage. Anesthesia and Analgesia. 98 (4), 1133 - 1139 (2004).

20. Roy, C.S. Sherrington, M.B. On the regulation of the blood-supply of the brain. The Journal of Physiology. 11 (1-2), $85-158$ (1890).

21. Mathew, B.P., DeWitt, D.S., Bryan, R.M., Bukoski, R.D., Prough, D.S. Traumatic brain injury reduces myogenic responses in pressurized rodent middle cerebral arteries. Journal of Neurotrauma. 16 (12), 177 - 186 (1999).

22. Bayliss, W.M. On the local reactions of the arterial wall to changes of internal pressure. The Journal of Physiology. 28 (3), 220 - 231 (1902). 
23. Johnson, P.C. Henrich, H.A. Metabolic and myogenic factors in local regulation of the microcirculation. Federation Proceedings. 34 (11), 2020 - 2024 (1975).

24. Johnson, P.C. The myogenic response and the microcirculation. Microvascular Research. 13 (1), 1 - 18 (1977).

25. Atkinson, C.L. et al. Opposing effects of shear-mediated dilation and myogenic constriction on artery diameter in response to handgrip exercise in humans. Journal of Applied Physiology. 119 (8), 858 - 864 (1985).

26. Johnson, P.C. The myogenic response in the microcirculation and its interaction with other control systems. Journal of Hypertension. Supplement. 7 (4), S33 - S39 (1989).

27. Allen, S.P., Wade, S.S., Prewitt, R.L. Myogenic tone attenuates pressure-induced gene expression in isolated small arteries. Hypertension. 30 (2 Pt 1), 203 - 208 (1997)

28. Owens, G.K., Kumar, M.S., Wamhoff, B.R. Molecular regulation of vascular smooth muscle cell differentiation in development and disease. Physiological Reviews. 84 (3), 767 - 801 (2004).

29. Ahn, D.S. et al.Enhanced stretch-induced myogenic tone in the basilar artery of spontaneously hypertensive rats. Journal of Vascular Research. 44 (3), 182 - 191 (2007).

30. Demay, J.G. Gray, S.D. Endothelium-dependent reactivity in resistance vessels. Progress in Applied Microcirculation. 8, 181 - 187 (1985).

31. Nilsson, H. Sjöblom, N. Distension-dependent changes in noradrenaline sensitivity in small arteries from the rat. Acta Physiologica Scandinavica. 125, 429 - 435 (1985).

32. Halpern, W. Kelley, M. In vitro methodology for resistance arteries. Blood Vessels. 28, 245 - 51 (1991).

33. Bryan, R.M.Jr. et al. Stimulation of $\alpha^{2}$ adrenoreceptors dilates the rat middle cerebral artery. Anesthesiology. 85, 82 - 90 (1996).

34. Golding, E.M. et al. Endothelial-mediated dilations following severe controlled cortical impact injury in the rat middle cerebral artery. Journal of Neurotrauma. 15 (8), 635 - 644 (1998).

35. Golding, E.M., Contant, C.F., Robertson, C.S., Bryan, R.M. Temporal effect of severe controlled cortical impact injury in the rat on the myogenic response of the middle cerebral artery. Journal of Neurotrauma. 15 (11), 973 - 984 (1998).

36. Golding, E.M. et al. Cerebrovascular reactivity to $\mathrm{CO}(2)$ and hypotension after mild cortical impact injury. The American Journal of Physiology. 277 (4 Pt 2), H1457 - H1466 (1999).

37. Speden, R.N. The use of excised, pressurized blood vessels to study the physiology of vascular smooth muscle. Experientia. 41,1026 - 1028 (1985).

38. Yu, G.X. et al. Traumatic brain injury in vivo. and in vitro. contributes to cerebral vascular dysfunction through impaired gap junction communication between vascular smooth muscle cells. Journal of Neurotrauma. 31 (8), 739 - 748 (2014).

39. Rodriguez, U.A. et al. Effects of mild blast traumatic brain injury on cerebral vascular, histopathological and behavioral outcomes in rats. Journal of Neurotrauma. 35 (2), 375 - 395 (2018).

40. Friedlander, F.G. The diffraction of sound pulses; diffraction by a semi-infinite plane. Proceedings of the Royal Society of London. Series A, Mathematical and Physical Sciences. 186 (1006), 322 - 344 (1946).

41. Tompkins, P. et al. Brain injury: neuro-inflammation, cognitive deficit and magnetic resonance imaging in a model of blast induced traumatic brain injury. Journal of Neurotrauma. 30 (22), 1888 - 1897 (2013).

42. Cipolla, M.J., Vitullo, L., McKinnon, J. Cerebral artery reactivity changes during pregnancy and the postpartum period: a role in eclampsia? American Journal of Physiology. Heart and Circulatory Physiology. 286 (6), H2127 - 2132 (2004).

43. Golding, E.M., Robertson, C.S., Bryan, R.M.Jr. Comparison of the myogenic response in rat cerebral arteries of different calibers. Brain Research. 785 (2), 293 - 298 (1998).

44. DeWitt, D.S. Prough, D.S. Assessment of cerebral vascular dysfunction after traumatic brain injury. In: Animal Models of Acute Neurological Injuries II: Injury and Mechanistic Assessments. Humana Press: New York (2012).

45. Degnan, A.J. et al. MR angiography and imaging for the evaluation of middle cerebral artery atherosclerotic disease. American Journal of Neuroradiology. 33 (8), 1427 - 1435 (2012).

46. Liu, Q. et al. Comparison of high-resolution MRI and CT angiography and digital subtraction angiography for the evaluation of middle cerebral artery atherosclerotic steno-occlusive disease. The International Journal of Cardiovascular Imaging. 29 (7), 1491 - 1498 (2013).

47. Newell, D.W. and Winn, H.R. Transcranial Doppler in cerebral vasospasm. Neurosurgery Clinics of North America. 1 (2), 319 - 328 (1990).

48. Schenone, M.H. Mari, G. The MCA Doppler and its role in the evaluation of fetal anemia and fetal growth restriction. Clinics in Perinatolgy. 38 (1), 83 - $102(2011)$

49. Morris, R.K., Say, R., Robson, S.C., Kleijnen, J., Khan, K.S. Systemic review and meta-analysis of middle cerebral artery Doppler to predict perinatal wellbeing. European Journal of Obstetrics, Gynecology and Reproductive Biology. 165 (2), 141 - 155 (2012).

50. Alford, P.W. et al. Blast-induced phenotypic switching in cerebral vasospasm. Proceedings of the National Academy of Sciences of the United States of America. 108 (31), 12705 - 12710 (2011). 